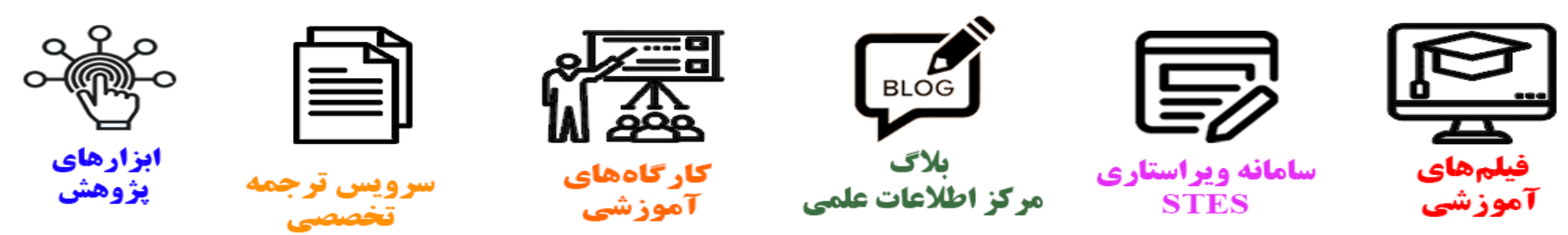

\title{
(c)
}

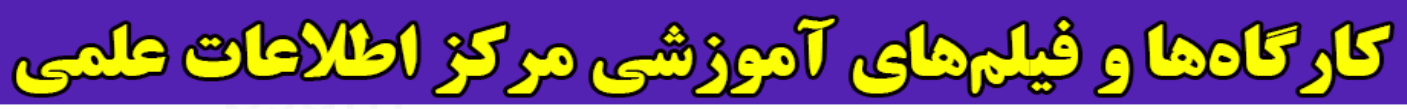
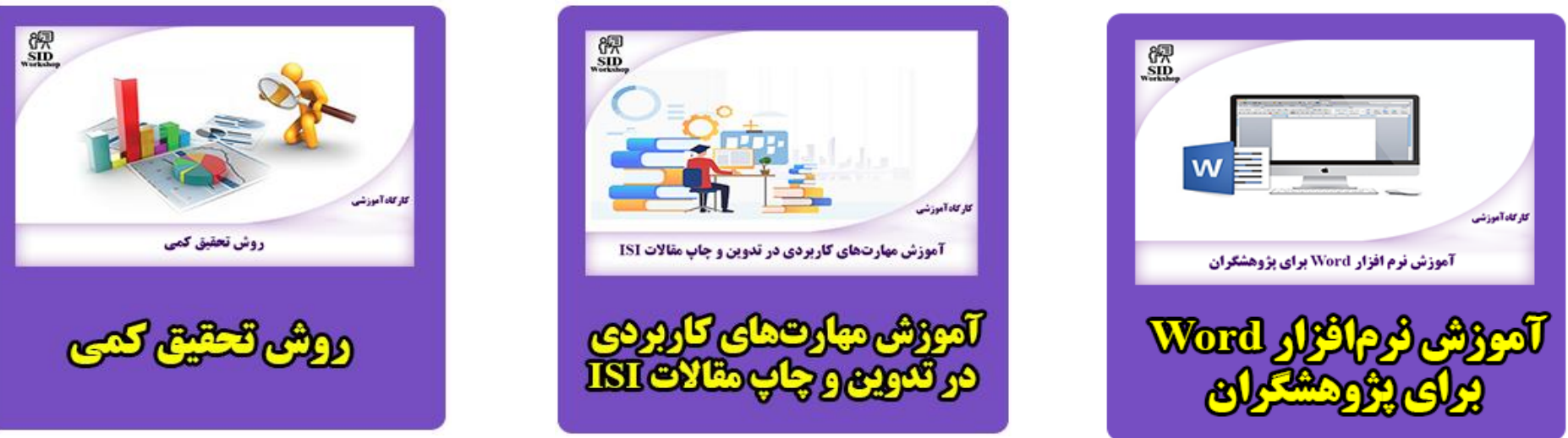


\title{
International Journal of Engineering
}

Journal Hom e page: ww w. i je.ir

\section{Lattice Boltzmann Simulation of Deformation and Breakup of a Droplet under Gravity Force Using Interparticle Potential Model}

\author{
S. E. Mousavi Tilehboni, K. Sedighi, M. Farhadi*, E. Fattahi
}

Faculty of Mechanical Engineering, Babol Noshirvani University of Technology, Postal Code 4714871167, Babol, Iran

\section{$P A P E R$ IN FO}

Paper history:

Received 16 January 2013

Received in revised form 17 February 2013

Accepted 28 February 2013

\section{Keywords:}

Falling droplet

Breakup

Lattice Boltzmann method

Interparticle potential model

Eotvos number

\section{$A B S T R A C T$}

In this paper interparticle potential model of the lattice Boltzmann method (LBM) is used to simulate the deformation and breakup of a falling droplet under the gravity force. First, this model is applied to ensure that the surface tension effect is properly implemented in this model. Two tests have been considered. First, it has been checked an initial square drop in a 2D domain can freely deform to a circular drop and secondly the coalescence of two static drops that merge to become a single circular drop is simulated. In order to further verify the model, Laplace law for static drops is performed. In the next step, wall effects on the droplet shape and its average velocity have been studied. It is seen that the average velocity of droplet at different times is independent of wall effects when the ratio of the width of the channel to droplet diameter (W/D) is more than 6. In the final section of the paper, deformation and breakup of a falling droplet for some range of Eotvos and Ohnesorge numbers are investigated. It is seen that at very low Eotvos numbers, where the surface tension force is dominant, the droplet deforms slowly and reaches a steady state without breakup. At higher Eotvos numbers gravitational force overcome the surface tension force and the droplet deforms more. For breakup modes at the small Ohnesorge number, if Eotvos number be increased to an intermediate value, the droplet deforms more than from a state of low Eotvos number value and eventually forms a backward-facing bag. Finally, for high Eotvos numbers, fragments of droplet are sheared from the edges and the shear breakup mechanism is seen. On the other hand, the stabilizing effect of the Ohnesorge number, (the ratio of viscous stresses and surface tension) is shown. At higher Ohnesorge number, the simulations show that the main effect of increasing Ohnesorge number is to move the boundary between the different breakup modes to higher Eotvos number.

\section{INTRODUCTION}

Deformation and breakup of a single droplet falling under gravitational forces is a crucial subject. The breakup mechanism of liquid droplets can be found in many industrial appliances such as fuel injectors, paint sprays, and ink - jet printers as well as in natural phenomena like raindrops, and is of great importance to applications such as mixing in multiphase systems, deformation of biological cells, blending of molten polymers, and, etc. There is a large body of work dealing with this subject under the general heading of two phase flows. Here, deformation and fragmentation of a single droplet falling under gravitational forces is of interest. Fakhri and Rahimian [1,2] presented numerical simulations of descending droplets for a variety of

\footnotetext{
* Corresponding Author Email:mfarhadi@nit.ac.ir (M. Farhadi)
}

Ohnesorge, Archimedes, and Eotvos numbers. All simulations were performed in $2 \mathrm{D}$ or axi-symmetric domains. Magarvey and Taylor [3] investigated the breakup of large falling drops in stagnant air by producing unstable droplets greater than about $7 \mathrm{~mm}$ in diameter. They observed that for the larger parent drop breakup is most likely to happen and the number of fragments tends to increase. Kojima et al. [4] studied deformation and breakup of a liquid droplet under gravity in another miscible fluid at low drop Reynolds numbers for low density and viscosity ratios. Ni et al. [5] investigated deformation and breakup of a falling droplet in a 2D channel with density ratios of 1.05 and 1.125. They solved the Navier-Stokes equations (NSE) using a projection method together with a level set technique. They also examined wall effects on the motion and the breakup of the drop. Feng [6] studied the steady state flow $\mathrm{f}$ deformable and outside of a 
deformable liquid drop falling at its terminal velocity using a Galerkin finite element method. Han and Tryggvason [7] studied the unsteady motion of falling liquid drops for different fluid-fluid combinations, using a finite difference front tracking method. Results are classified for two density ratios, 1.5 and 10, and different Eotvos and Ohnesorge numbers. Jalaal and Mehravaran [8] using the direct numerical simulation, studied the deformation, disintegration and dispersion of fragments of a single falling droplet.

Recently, the lattice Boltzmann method (LBM) has been developed from the lattice-gas automata $[9,10]$. The LBM has emerged as an alternative method for conventional computational schemes. Unlike conventional numerical methods based on the discretization of macroscopic continuum equations, the LBM is based on microscopic models and mesoscopic kinetic equation. The LBM recovers the Navier-Stokes equations in the incompressible flow limit. This model is a power full method for simulation of single phase flows, such as dispersion and deposition of micro particles [11], nanofluid [12], nano-particles [13], porous media [14] and etc.

The LBM is a promising technique in modelling multiphase flow problems, particularly for flows with complex topological changes of the interface or in complex geometries. In recent years, it has been exposed to the attention of researchers due to the lower run-time compared with other CFD methods, simplicity of applying the multiphase flow equations in the LBM algorithm, and capability of LBM in solving unsteady problems. A number of models have been developed to model multiphase flows in LBM. The color function model Proposed by Gunstensen et al. [15] is the first multiphase model in LBM. Two components are used in this model to represent two types of fluids. The red and blue particle distributions each follow its own LB equation. The collision term in the equation includes both the self-interactions of particles of the same type, and the cross interactions of particles with different colors. The latter one is used to generate a surface tension between the two phases, and is calculated using the gradient of the color function.

In order to maintain phase segregation, the particle density near the interface needs to be redistributed to minimize mixing. The interparticle potential model proposed by Shan and Chen [16] is the most widely used multiphase LBM model due to its simplicity and versatility, and for this study due to simplicity of taking place external force in the model because of existence separate forces term, and capability of model for simulating flows at small Ohnesorge numbers $(\mathrm{Oh}<0.1)$. An interaction force between the particles is introduced to account for the molecular interactions in non-ideal fluids. Consequently, the fluid spontaneously segregates into dense and dilute phases under proper condition according to the equation of state of the fluid. The automatic phase separation is an attractive feature in this model, since it does not require the interface-tracking or interface-capturing step that is often necessary in other models. The implementation of the model is also straight forward since the interaction force can be simply calculated from the pair-wise interaction potential. In recent years, many researches have been conducted to discuss the properties and the improvements of the interaction potential model (Shan, [17]; Sbragaglia et al., [18]). The free energy approach proposed by Swift et al. [19] is based on the thermodynamic equilibrium of fluid phases. The free energy functional at a fixed temperature includes both the bulk part and the interface part. The former introduces equilibrium between two phases, while the latter introduces surface tension. The model is incorporated into LBE by adding a term to the equilibrium distribution to ensure the correct stress tensor condition. The free energy model is also capable for automatic phase separation, and it is easier to specify density and surface tension values in the free energy model than in the interaction potential model. However, a major criticism of the free energy model is that it lacks Galilean invariance, i.e. the physical properties in the model are dependent upon the velocity. Proposed by $\mathrm{He}$ et al. $[20,21]$, the mean-field theory treats the interparticle interaction using the mean-field approximation. The mean-field theory model uses two sets of particle distribution function (pdfs) to simulate the hydrodynamic properties. The first set of pdf describes pressure and velocity fields, whereas the second set tracks the dynamics of the interface.

In this paper, the multiphase model proposed by Shan and Chen (S-C model) [16] is employed to investigate the deformation and breakup of a droplet falling due to the gravitational effects. There are many studies which applied this model in the two-phase flow problems. However, in this study for the first time S-C model has been used to simulate falling droplet in the vertical channel and first time lattice Boltzmann method is applied to investigate the deformation and breakup of droplet under gravity at small Ohnesorge number $(\mathrm{Oh}<0.1)$. In fact, this study demonstrated the ability of $\mathrm{S}-\mathrm{C}$ model in the LBM for simulated falling droplet at small Ohnesorge numbers. In the previous studies of falling droplet in 2D vertical channel by LBM, dynamic behaviour of the droplet at small Ohnesorge number $(\mathrm{Oh}<0.1)$ has not been studied. In this study, the side wall effects have been studied on falling droplet in the elongated vertical channel by LBM for the first time.

In the next sections of this paper, following subjects are studied: In Section 2, the governing equations and methodology of the S-C model of the lattice Boltzmann method are given. In section 3, this model validation is presented. Section 4 represents the numerical results of deformation and breakup of a falling droplet. Finally, a summary of the results and conclusions are given. 


\section{SHAN AND CHEN INTERPARTICLE POTENTIAL LBE MODEL}

2. 1. Description of the Model the Interparticle potential model of the lattice Boltzmann method proposed by Shan and Chen [16] is applied to study droplet falling in the vertical channel under gravity force. Shan and Doolen [22] have reviewed this multiphase model and the details of the multiple component LBE model with interparticle forces are given. For a 2D vertical channel, simulations are conducted on D2Q9 [23] lattice. The inteparticle potential model can be applied to simulate a binary mixture. In this case, each component $\sigma(\sigma=1$ or 2$)$ has its own distribution functions $f_{i}^{\sigma}$ that are governed by their own evolution equations:

$f_{i}^{\sigma}\left(\boldsymbol{x}+\boldsymbol{c}_{i} \delta_{t}, t+\delta_{t}\right)-f_{i}^{\sigma}(\boldsymbol{x}, t)=-\frac{1}{\tau^{\sigma}}\left(f_{i}^{\sigma}(\boldsymbol{x}, t)-f_{i}^{\sigma(e q)}\left(n^{\sigma}, \boldsymbol{u}_{e q}^{\sigma}\right)\right)$

where, $f_{i}^{\sigma}(x, t)$ is the number density distribution of component $\sigma$ with velocity $\mathrm{c}_{\mathrm{i}}$ at position $\boldsymbol{x}$ and time $t$. $\tau^{\sigma}$ is the relaxation parameter of component $\sigma$ and $f_{i}^{\sigma(e q)}$ is the equilibrium distribution given by:

$f_{i}^{\sigma(e q)}=\omega_{i} n^{\sigma}\left[1+\frac{\boldsymbol{c}_{i} \cdot \boldsymbol{u}_{e q}^{\sigma}}{c_{s}^{2}}+\left(\frac{\boldsymbol{c}_{i} \cdot \boldsymbol{u}_{e q}^{\sigma}}{2 c_{s}^{4}}\right)^{2}-\frac{\boldsymbol{u}_{e q}^{\sigma 2}}{2 c_{s}^{2}}\right]$

Discrete lattice velocities in the above equation are

$\left[c_{1}, c_{2}, c_{3}, c_{4}, c_{5}, c_{6}, c_{7}, c_{8}\right]=\left[\begin{array}{ccccccccc}0 & 1 & 0 & -1 & 0 & 1 & -1 & -1 & 1 \\ 0 & 0 & 1 & 0 & -1 & 1 & 1 & -1 & -1\end{array}\right]$

and weighting factors are

$w_{i}= \begin{cases}\frac{4}{9}, & \mathrm{i}=0 \\ \frac{1}{9}, & \mathrm{i}=1,2,3,4 \\ \frac{1}{36}, & \mathrm{i}=5,6,7,8\end{cases}$

Relaxation parameter $\left(\tau^{\sigma}\right)$ related to the kinematic viscosity by:

$\tau^{\sigma}=c_{s}^{2}\left(\tau^{\sigma}-0.5 \delta t\right)$

where, $v^{\sigma}$ is kinematic viscosity of component $\sigma . \delta t=1$ and $c_{s}^{2}=R T=\frac{1}{3}$ are chosen, where $\mathrm{c}_{\mathrm{s}}$ is speed of sound in Lattice scale. The number density and velocity of component $\sigma$ can be obtained by:

$n^{\sigma}(\boldsymbol{x}, t)=\sum_{i} f_{i}^{\sigma}(\boldsymbol{x}, t)$
$n^{\sigma} \boldsymbol{u}^{\sigma}(\boldsymbol{x}, t)=\sum_{i} \boldsymbol{c}_{i} f_{i}^{\sigma}(\boldsymbol{x}, t)$

The mass density is

$\rho^{\sigma}=m^{\sigma} n^{\sigma}$

where, $m^{\sigma}$ is the molecular mass of component $\sigma$.
In order to induct nonlocal interaction among particles, Shan and Chen [16] defined an interaction potential between components $\sigma$ and $\bar{\sigma}$

$V\left(\boldsymbol{x}, \boldsymbol{x}^{\prime}\right)=G_{\sigma \bar{\sigma}} \psi^{\sigma}(\boldsymbol{x}) \psi^{\sigma}\left(\boldsymbol{x}^{\prime}\right)$

where, $\boldsymbol{x}^{\prime}=\boldsymbol{x}+\boldsymbol{c}_{i} \delta t$ is the location of the neighbor sites, $\psi^{\sigma}$ and $\psi^{\bar{\sigma}}$ denotes the interaction potential of components $\sigma$ and $\bar{\sigma}$, respectively. $G_{\sigma \bar{\sigma}}$ is the interaction strength between two components $\sigma$ and $\bar{\sigma}$. For two immiscible fluids, when $\sigma$ and $\bar{\sigma}$ are different (denoting two different phases), $G_{\sigma \bar{\sigma}}$ is positive, resulting in repulsion forces between the particles of the two phases. When $\sigma$ and $\bar{\sigma}$ are the same, Equation (8) presents the interaction between the particles of one phase, which implies that the nature of the force is attractive and therefore $G_{\sigma \bar{\sigma}}$ should have a negative value. The magnitude of $G_{\sigma \bar{\sigma}}$ controls the surface tension. For nearest neighbor interactions, $G_{\sigma \bar{\sigma}}$ is defined as:

$G_{\sigma \bar{\sigma}}\left(\boldsymbol{x}, \boldsymbol{x}^{\prime}\right)= \begin{cases}0 & \left|\boldsymbol{x}-\boldsymbol{x}^{\prime}\right|>\left|\boldsymbol{c}_{i} \delta t\right| \\ G_{\sigma \bar{\sigma}} & \left|\boldsymbol{x}-\boldsymbol{x}^{\prime}\right|=\left|\boldsymbol{c}_{i} \delta t\right|\end{cases}$

The interaction strength parameters $G_{11}$ and $G_{22}$ describe the interaction within each individual component. The original form of the interaction potential introduced by Shan and Chen [16, 24] is widely used and is given as follows:

$\psi(\rho)=\rho_{0}\left(1-\exp \left(\frac{-\rho}{\rho_{0}}\right)\right)$

In Equation (10), $\rho_{o}$ was set equal to 1 . While component 2 is assumed to be ideal gas so that $\mathrm{G}_{22}=0$ and $\psi(\rho)=\rho$ [24-26], the interaction between the two components and the immiscibility of the mixture are described by $\mathrm{G}_{12}$.

In the multicomponent model proposed by Martys and Chen [27], the macroscopic velocity $\left(\boldsymbol{u}_{e q}^{\sigma}\right)$ is given by

$\boldsymbol{u}_{e q}^{\sigma}=\boldsymbol{u}^{\prime}+\frac{\tau^{\sigma} \boldsymbol{F}^{\sigma}}{\rho^{\sigma}}$

where, $\boldsymbol{u}^{\prime}$ is a velocity common to the various components defined as

$\boldsymbol{u}^{\prime}=\frac{\sum_{\sigma} \rho^{\sigma} \boldsymbol{u}^{\sigma} / \tau^{\sigma}}{\sum_{\sigma} \rho^{\sigma} / \tau^{\sigma}}$

and $\boldsymbol{F}^{\sigma}$ is the total interaction force on fluid component $\sigma$ including fluid-fluid interaction $\left(\boldsymbol{F}_{f}^{\sigma}\right)$ fluid-solid interaction $\left(\boldsymbol{F}_{s}^{\sigma}\right)$ and gravity force $\left(\boldsymbol{F}_{g}^{\sigma}\right)$, i.e.:

$\boldsymbol{F}^{\sigma}=\boldsymbol{F}_{f}^{\sigma}+\boldsymbol{F}_{s}^{\sigma}+\boldsymbol{F}_{g}^{\sigma}$ 
Given the form of potential in Equation (8), the rate of net momentum change induced at each site due to the interaction is simply given as in [16]:

$\boldsymbol{F}^{\sigma}=\frac{d p^{\sigma}(\boldsymbol{x})}{d t}=-\nabla V\left(\boldsymbol{x}, \boldsymbol{x}^{\prime}\right)=-\psi^{\sigma}(\boldsymbol{x}) \sum_{\sigma} G_{\sigma \bar{\sigma}} \sum_{i} \psi^{\bar{\sigma}}\left(\boldsymbol{x}+\boldsymbol{c}_{i} \delta t\right) \boldsymbol{c}_{i}$

In case of the fluid-solid wall interaction, separate forces should be calculated for each phase to account for the fluid-solid forces. T, he source term has the same formulation for both single component multiphase [28] and multicomponent multiphase flows [27], namely:

$\boldsymbol{F}_{s}^{\sigma}=-\psi^{\sigma}(\boldsymbol{x}) \sum_{i} G_{a d s}^{\sigma} w_{i} S\left(\boldsymbol{x}+\boldsymbol{c}_{i} \delta_{t}\right) \boldsymbol{c}_{i}$

where, $G_{a d s}^{\sigma}$ is the adsorption coefficient for phase $\sigma$, and $w$ is the weighting factor as Equation (4) for the D2Q9 lattice, $S$ is a switch, which takes on the value 1 when the lattice node at $\boldsymbol{x}+\boldsymbol{c} \delta t$ is a solid node, otherwise it is zero, for the present study, the solid nodes are not moving and therefore $S$ is not a function of time. Interaction of the solid and fluid nodes determines the contact angle of the liquid and solid surface, the adsorption coefficient, $G_{\text {ads }}^{\sigma}$ determines the magnitude and type of the interaction force. Based on the magnitude of the adsorption coefficient, three different types of surface can be specified, namely: hydrophobic (non wetting), neutral, and hydrophilic (wetting) surfaces [27, 29].

Finally, the macroscopic densities and velocity are calculated from the distribution functions:

$$
\begin{aligned}
& \rho(\boldsymbol{x}, t)=\sum_{\sigma} m^{\sigma} \sum_{i} f_{i}^{\sigma}(\boldsymbol{x}, t) \\
& \rho(\boldsymbol{x}, t) \boldsymbol{U}(\boldsymbol{x}, t)=\sum_{\sigma} m^{\sigma} \sum_{i} \boldsymbol{c}_{i} f_{i}^{\sigma}(\boldsymbol{x}, t)+\frac{1}{2} \sum_{\sigma} \boldsymbol{F}^{\sigma}(\boldsymbol{x}, t)
\end{aligned}
$$

and function of total pressure are obtained [30,31].

$$
P(\boldsymbol{x}, t)=R T \sum_{\sigma} n^{\sigma}+\frac{R T}{2} \sum_{\sigma \bar{\sigma}} G_{\sigma \bar{\sigma}} \psi^{\sigma} \psi^{\bar{\sigma}}
$$

2. 2. Gravity Force Falling of a droplet is the result of gravity force and density difference between two fluids (components or phases). Figure 1 shows the schematic of gravity and buoyancy effects on droplet suspended in the other fluid. In order to consider the buoyant effect associated with density difference between two fluids, an effective buoyant force, $\left(\boldsymbol{F}^{e, \sigma}\right)$ was introduced. It is defined by [26]

$\boldsymbol{F}^{e, \sigma}=\rho^{\sigma} \times \boldsymbol{g}_{\text {applied }}$

In fact, because of the density difference between the phases, the buoyancy and gravity Consequence is defined as:

$$
\boldsymbol{F}_{\text {net,g }}=\boldsymbol{g}_{\text {eff }} \Delta \rho_{\text {eff }}=\boldsymbol{g}_{\text {eff }}\left(\rho_{H}-\rho_{L}\right)
$$

Equating Equations (18) and (19) yields to:

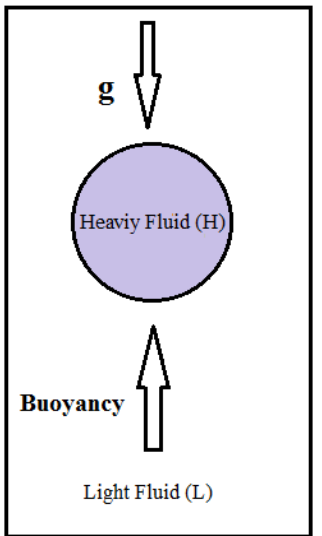

Figure 1. Representation of the buoyancy force versus the weight force. $\rho_{H}$ is the heavier fluid density (droplet fluid in this study), and $\rho_{L}$ the lighter fluid density (surrounding fluid in this study).

$\boldsymbol{g}_{\text {applied }}=\frac{\boldsymbol{g}_{e f f}\left(\rho_{H}-\rho_{L}\right)}{\rho_{\sigma}}$

where, $\rho_{H}$ is the heavier fluid density and $\rho_{L}$ is the lighter fluid density, and $\rho_{\sigma=H}$ is the density of the droplet as actually used in the simulation. The $\boldsymbol{g}_{\text {applied }}$ represents the gravitational acceleration actually used in the simulation and applied to component $\sigma$ only. Thus, $\Delta \rho_{\text {eff }}$ and $\boldsymbol{g}_{\text {eff }}$ are the Effective physical density difference and gravitational acceleration quantities. While the $\rho_{\sigma=H}$ and $\boldsymbol{g}_{\text {applied }}$ are simply simulation surrogates that yield the same buoyancy effect. This approach of placing the buoyant effects into a body force greatly expands the range of effective density difference between droplet and surrounding fluid that can be readily simulated with the S-C model.

\section{NUMERICAL VALIDATION}

3. 1. Relaxation of Liquid Drops To ensure that the surface tension effect is properly implemented in this model, two test cases is considered. First, it has been checked an initial square drop in a 2D domain can freely deform to a circular drop (see Figure 2) and secondly the coalescence of two static drops that merge to become a single circular drop is simulated (see Figure 3 ). In the first test, physical setup is a square drop with a length of 40 lattice units which is placed in the middle of the computational domain with $100 \times 100$ lattice units. In the second test, physical setup is two circular drops with Radius 15 lattice units which is placed in the computational domain with. $160 \times 160$ lattice units and in both of them periodic boundary conditions are applied in all directions. In all simulations for this paper, $\rho_{\mathrm{L}=\mathrm{c}}=0.25, \rho_{\mathrm{H}=\mathrm{d}}=1$ ('c' denotes the continuous or 
surrounding fluid and 'd' denotes droplet fluid). Thus, the density ratio $\left(\rho_{d} / \rho_{c}\right)$ is 4 . For both fluids the kinematic viscosity is 0.1667 .

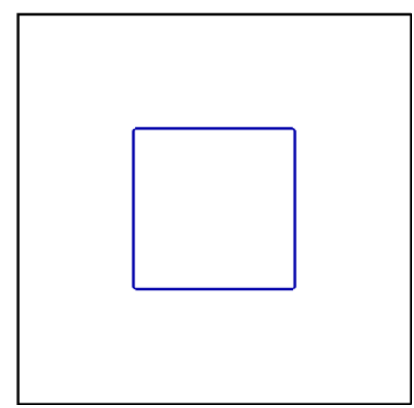

$\mathrm{T}=0$

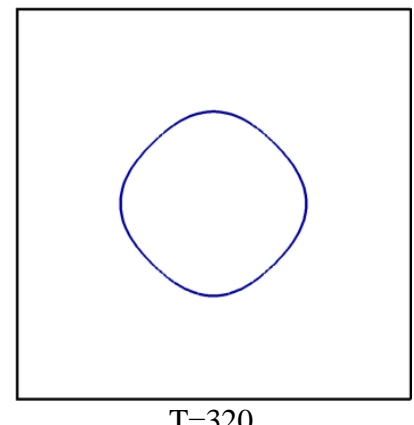

$\mathrm{T}=320$

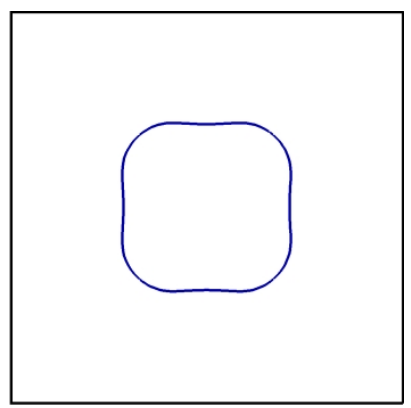

$\mathrm{T}=80$

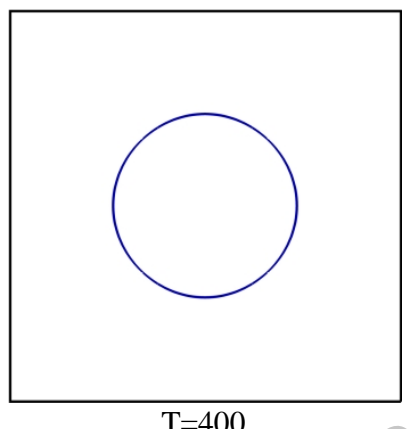

$\mathrm{T}=400$
Both cases show deformation to the final circular drop, indicating that the surface tension effect is correctly implemented.

Figure 2. Free deformation of a static drop from square shape to a circular shape at different lattice time steps (iterations of LBM).

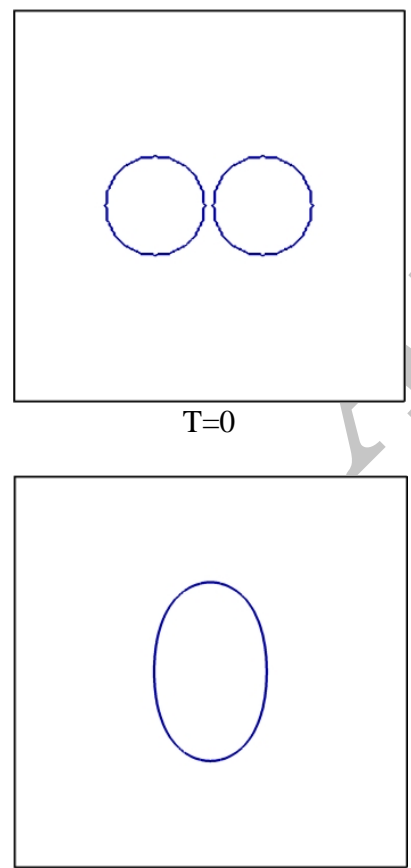

$\mathrm{T}=1600$

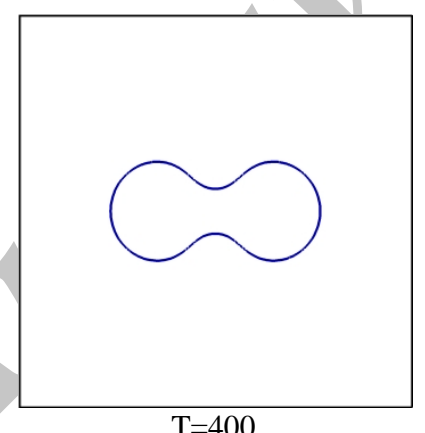

$\mathrm{T}=400$

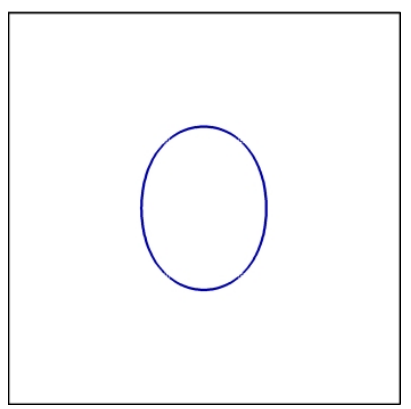

$\mathrm{T}=2000$

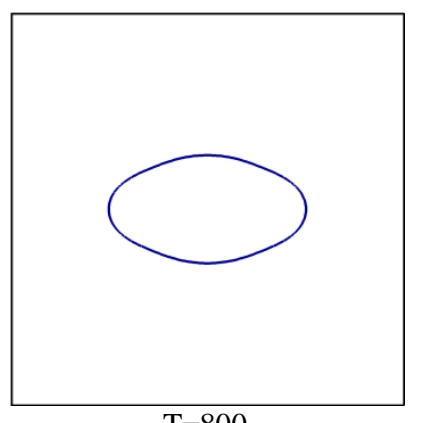

$\mathrm{T}=800$

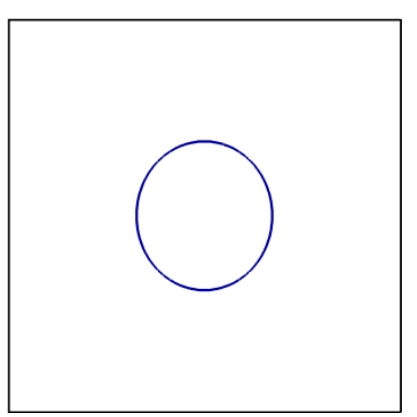

$\mathrm{T}=4000$

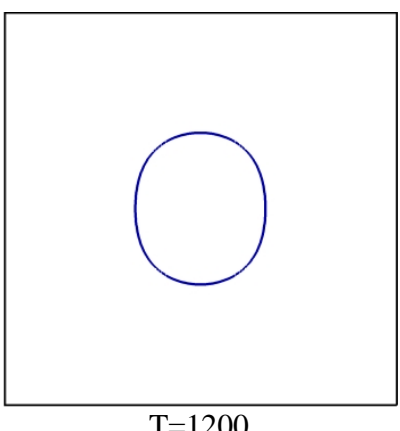

$\mathrm{T}=1200$

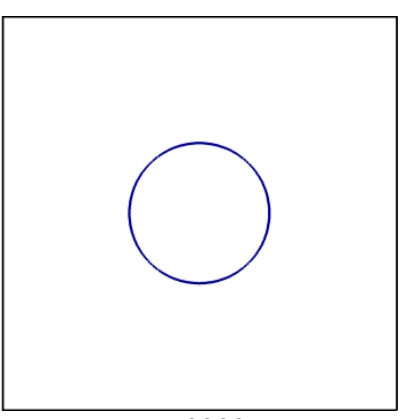

$\mathrm{T}=8000$

Figure 3. Coalescence of two identical circular drops and free deformation to a circular shape at different lattice time steps (iterations of LBM). 
3. 2. Laplace law To further validate the code, the static drop test is performed. The interfacial tension was evaluated based on Laplace's equation given by the following expression [32]:

$$
\Delta P=P_{\text {in }}-P_{\text {out }}=\frac{\sigma}{R}
$$

where, $R$ is the radius of the drop at equilibrium and $\sigma$ is the surface tension coefficient, and $\Delta P$ is the pressure difference between the inside and the outside of the drop. In order to verify the Laplace law, initially static drops with different radii are generated inside the computational domain with $100 \times 100$ lattice units. The domain boundary used periodic boundary conditions. The pressure difference was calculated using equation of state in Equation (17). The predicted values of the pressure difference are plotted against $1 / R$ in Figure 4. Figure 4 presents the pressure difference between the inside and the outside of a series of drops of different sizes as a function of the inverse drop radius. It can be seen that the Laplace's law is satisfied, because the linear fit of the points on the plot is in very good agreement with points. The linear relationship between $\Delta P$ and the inverse of the drop radius confirms a constant value of the surface tension. The slope of the linear line is 0.063 representing the coefficient of surface tension between two fluids for this study. Figure 5 shows the density profile along the centerline of a static drop, as indicated the density ratio, $\rho_{H} / \rho_{L}=4$ and $\mathrm{R}=25$ lattice units.

\section{RESULTS}

In this section, the main objective of the paper is delved, i.e. simulation and discussion of the falling droplet under gravitational effects. For all subsequent simulations, bounce-back boundary condition (BC) is applied at the side walls and periodic BC is used at the up and down boundaries [33].

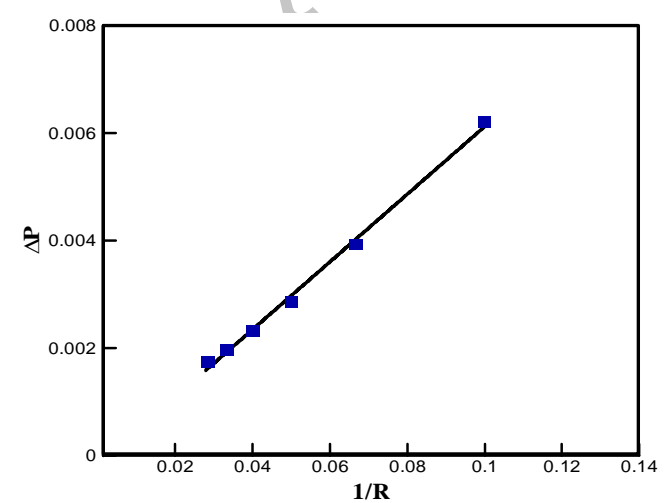

Figure 4. Verification of Laplace law. Pressure difference between the inside and the outside of a series of drops as a function of inverse drop radius with linear fit of the points.

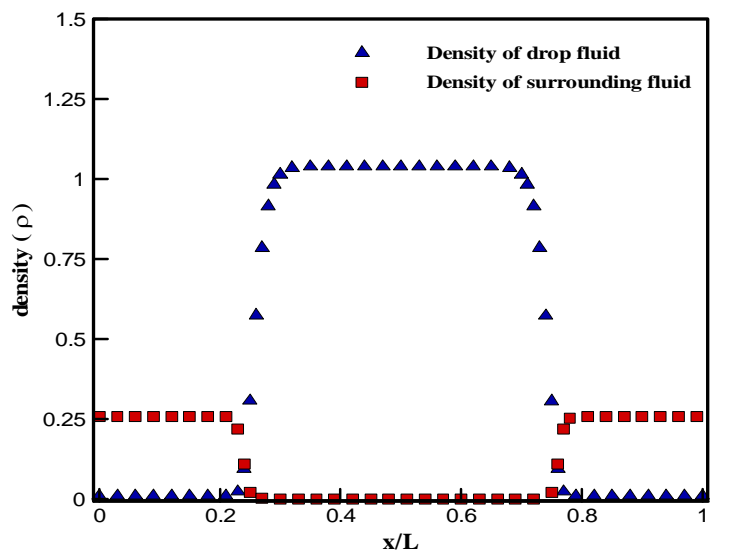

Figure 5. Density profile along the centerline of the drop. The heavier Liquid (drop fluid) is surrounded by the light fluid.

4. 1. Non-dimensional Numbers In the case of a falling droplet in a motionless media, the acceleration due to gravity, surface tension between the fluids, viscosity of fluids, initial diameter of the droplet and density of fluids are the main parameters. Considering these parameters, different non-dimensional groups governing the behavior of the droplet are proposed by researchers. A list of important dimensionless numbers for this study is as follows:

Eotvos number: $E o=\frac{\mathrm{g}\left(\rho_{d}-\rho_{c}\right) D^{2}}{\sigma}$
Ohnesorge number: $O h=\frac{\mu_{d}}{\left(\rho_{d} D \sigma\right)^{1 / 2}}$

(based on droplet properties)

Ohnesorge number: $O h=\frac{\mu_{c}}{\left(\rho_{c} D \sigma\right)^{1 / 2}}$

(based on continuous fluid properties)

Dimensionless time $: t^{*}=\frac{T}{(D / g)^{\frac{1}{2}}}$

Reynods number : $\operatorname{Re}=\frac{V_{\text {ave }} D}{v_{d}}$

(based on droplet properties)

(Morton number) : Mo $=\frac{g\left(\rho_{d}-\rho_{c}\right) \rho_{c}{ }^{2} v_{c}^{4}}{\sigma^{3}}$

where, $\mathrm{g}$ is the gravitational acceleration, $\mathrm{D}$ is the initial diameter of the drop, $\rho_{d}$ and $\mu_{d}$ are density and viscosity of the drop, $\rho_{c}$ and $\mu_{c}$ are density and viscosity of the continuous phase, respectively, and $v_{d}$ and $v_{c}$ are kinematic viscosities of droplet and surrounding fluid, respectively, $\mathrm{T}$ is the lattice time step (iterations of LBM) and $\mathrm{V}_{\text {ave }}$ is the average velocity of droplet at any time. Since the Ohnesorge numbers are interdependent, in this paper results are presented based on $\mathrm{Oh}_{\mathrm{d}}$ and consequently obtained $\mathrm{Oh}_{\mathrm{c}}$.Hence, The $\mathrm{Oh}_{\mathrm{c}}$ will be changed by changing the $\mathrm{Oh}_{\mathrm{d}}$. 
4. 2. Correlation with Other Works Annaland et al. [34] studied the dynamic behavior of co-axial two initially spherical bubbles using the volume of fluid (VOF) method. In this section, before the study of falling droplet in the vertical channel, rising of two co axial bubbles with initially circular shape has been shown. Simulations are performed for $E O=16$ and $M o=2 \times 10^{-4}$. Figure 6 compares the results of this study with those obtained by Annaland et al. [34]. As in the case of co-axial bubbles, the leading bubble seems to freely rise in the surrounding liquid. The trailing bubble is affected by the leading bubble, and experiences more deformation when it enters the wake region behind the leading bubble. The difference between the two methods might be the result of the dimensions of the system and the difference in the density and viscosity ratios. Despite this difference in shape deformation, simulations of this study for bubbles shape are qualitatively agree well with the result of Annaland et al. [34] (Figure 6).
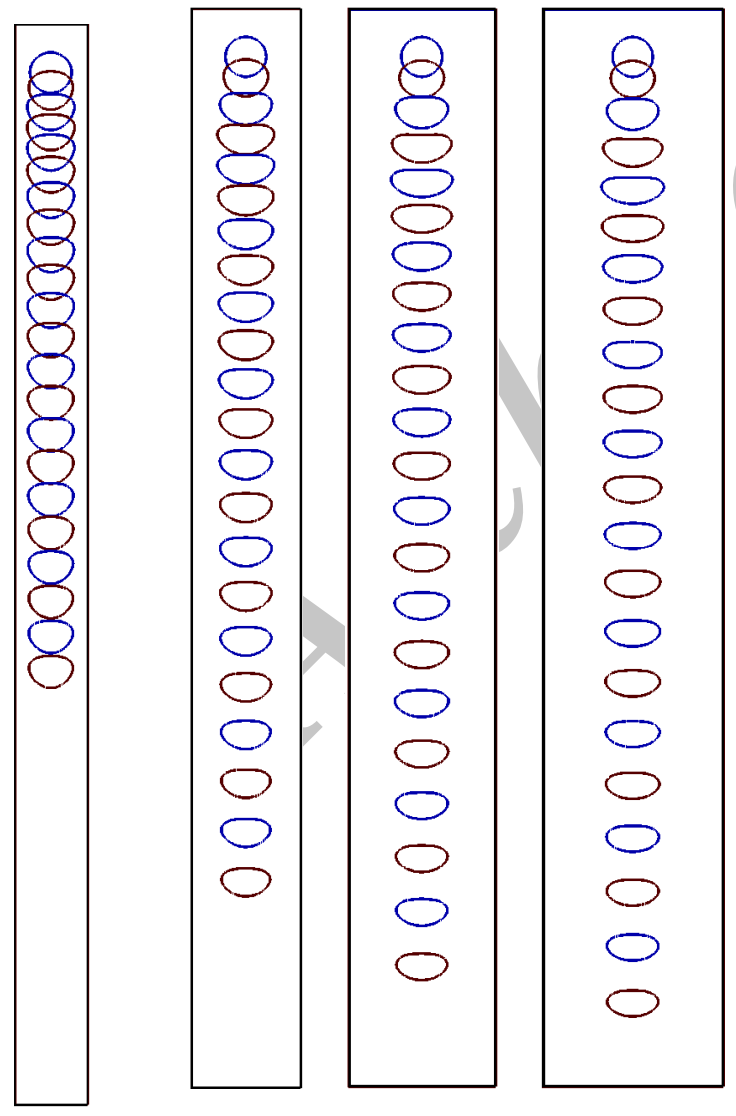

(a)

(b)

(c)

(d)

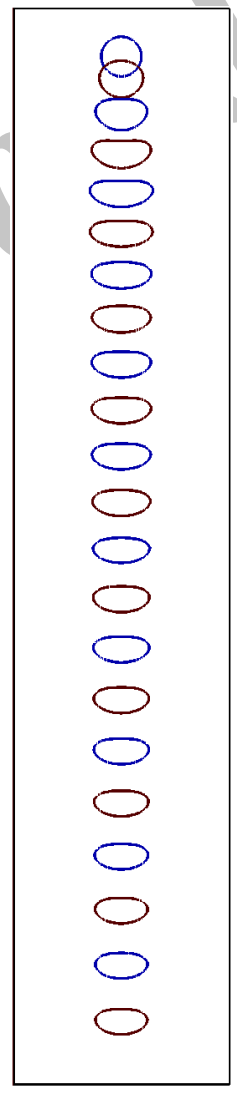

(e)

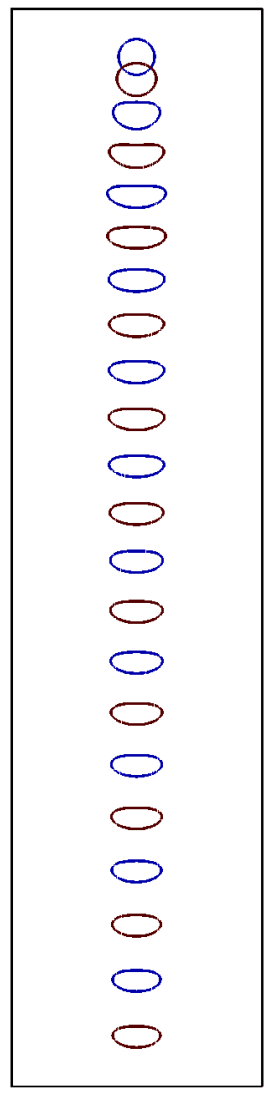

(f)

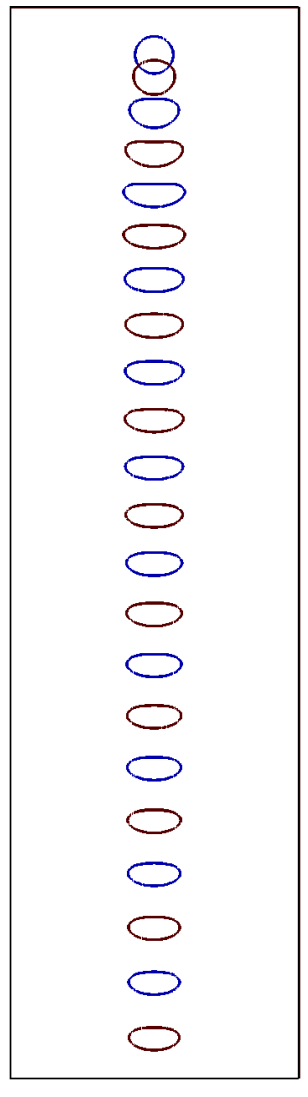

(g)

Figure 7. Snapshots of deformation of a falling droplet in domains with different ratios of W/D, (the first shape in each frame corresponds to $\mathrm{t}^{*}=0$, for all cases $\Delta \mathrm{t}^{*}=1$ and the last shape is plotted at $\mathrm{t}^{*}=21, \mathrm{Eo}=5, \mathrm{Oh}_{\mathrm{d}}=0.105$ and $\mathrm{Oh}_{\mathrm{c}}=0.053$, (a) W/D=2, (b) W/D=3, (c) $\mathrm{W} / \mathrm{D}=4$, (d) $\mathrm{W} / \mathrm{D}=5$, (e) $\mathrm{W} / \mathrm{D}=6$, (f) $\mathrm{W} / \mathrm{D}=7$, (g) $\mathrm{W} / \mathrm{D}=8$ 

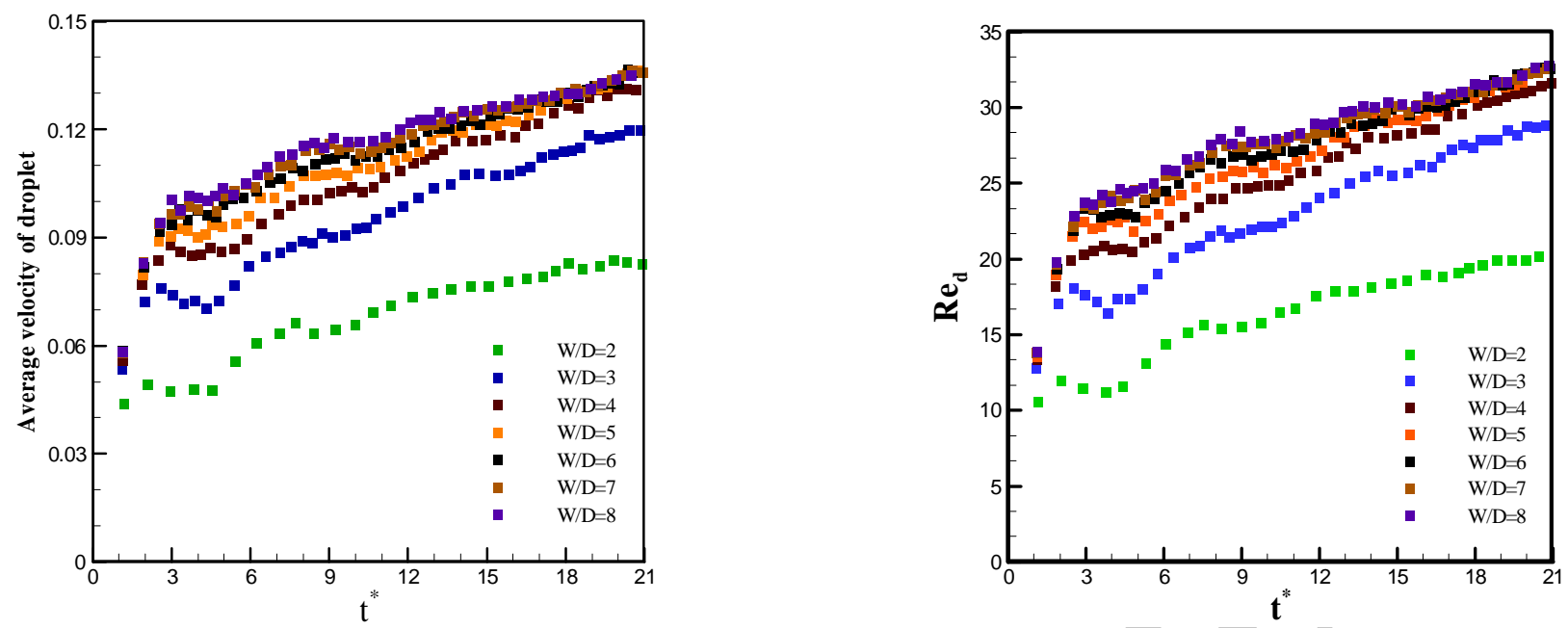

Figure 8. Wall effects on average velocity and Reynolds number, (a) Average velocity of droplet (b) Reynolds number of droplet, versus dimension-less time for domains with different ratios of $\mathrm{W} / \mathrm{D}$, for all cases $\mathrm{Eo}=5, \mathrm{Oh}_{\mathrm{d}}=0.105$ and $\mathrm{Oh}_{\mathrm{c}}=0.053$

TABLE 1. Terminal average velocity and Reynolds number of droplet for different ratios as W/D.

\begin{tabular}{cccccccc}
\hline$\frac{W}{D}$ & 2 & 3 & 4 & 5 & 6 & 7 & 8 \\
\hline$V_{\text {ave }}$ & 0.08308 & 0.118969 & 0.129680 & 0.132805 & 0.133381 & 0.133665 & 0.134284 \\
$\operatorname{Re}_{d}$ & 19.9359 & 28.5471 & 31.1170 & 31.8670 & 32.0052 & 32.0734 & 32.2218 \\
\hline
\end{tabular}

4. 3. Wall Effects on Shape and Velocity of Droplet on the Falling In a domain with side walls, droplet shape and falling velocity are affected by the walls of the domain. The magnitude of these effects depends on the ratio of the droplet diameter (D) to the width of the domain (W). In order to quantify the wall effects, an ellipsoidal regime of droplet where, $E o=5$ and $O h_{d}=$ 0.105 is considered for this investigation, various ratios for width of the domain to the droplet diameter (W/D) are chosen. Figure 7 displays the effect of these ratios on droplet shape and Figure 8 Shows these effects on the average falling velocity and Reynolds number of the droplet at different times. As shown in Figure 8, as the ratio of W/D increase, the wall effect on the droplet behavior becomes less significant. According to Table 1 , for ratio of $\mathrm{W} / \mathrm{D}=6$ and 7 , difference between terminal Reynolds number is less than $0.3 \%$. Thus, it is can be assumed that average velocity of droplet at different times is independent of wall effects when the ratio of W/D is more than 6. Figure 9 shows terminal Reynolds number versus W/D where $6<\mathrm{W} / \mathrm{D}$, approximately desire to a constant value. Accordingly to all next simulations in this paper, $\mathrm{W} / \mathrm{D}=6$. is chosen.

\section{4. Effect of Eo at Small Oh in the Falling Droplet}

When Ohnesorge number is small and surface tension is much more important than viscous stresses, Ohnesorge number has little influence on the breakup and Eotvos number is the main controlling parameter. Here, results are present for different Eotvos numbers While the Ohnesorge number is small $(\mathrm{Oh}<1)$. When a droplet is set into motion by a constant body force (gravity), the hydrodynamics pressure is higher at the poles and lower at the equator and the droplet deforms into an oblate ellipsoid. This deformation is opposed by the surface tension. Depending on the relative strength of the pressure forces and the surface tension, measured by Eotvos number, different breakup modes are observed. Based on these results, the evolution of droplets with $\rho_{\mathrm{d}} / \rho_{\mathrm{c}}=4$ at a small Ohnesorge number can be classified into four kinds in order of increasing Eotvos number: steady deformation, formation of a backward-facing bag and then breakup, oscillating indented droplet, and shearing of a film from the edge of the droplet and thus breakup. In Figure 10, the evolution of a droplet with a density ratio $\rho_{d} / \rho_{c}=4$, is shown for different Eotvos numbers. Values of Ohnesorge numbers, $\mathrm{Oh}_{\mathrm{d}}=0.074$ and $\mathrm{Oh}_{\mathrm{o}}=0.037$ are chosen, so that viscous stresses are low compared to surface tension. It is evident from Figure 10 that droplets breaking up in the backward- 
facing mode travel a much longer distance than those breaking up in the shear breakup mode.

In addition, note that for the same breakup mode, the rate of droplet deformation increases as Eotvos number increases. When Eotvos number is very low, the droplet deforms into an oblate ellipsoid and moves with a steady state shape as shown in Figure 10a for Eo=2. When Eotvos number is increased to 6 in Figure 10b, the droplet deformation is more pronounced. Initially, the droplet assumes a shape similar to that shown in Figure 10a, but then the back of the droplet becomes increasingly more convex and eventually the droplet deforms into a thin disk-like shape that moves at a nearly steady state. When Eotvos number is increased to 12 in Figure 10c, the droplet deforms more and eventually forms a backward-facing bag. Where Eotvos number is 24 (Figure 10d), the droplet moves with an essentially steady convex shape, showing no sign of bag formation.

At Eo $=48$ in Figure 10e and 11, initial stages of deformation are similar to the previous cases, but at final stages the droplet becomes slender at the poles. Ultimately, fine fragments of droplet are sheared from the edges and it can be seen the shear breakup mechanism. The type of breakup in Figure 10f and 12 at Eo $=72$ is the same as previous case with Eo $=48$. However, the rate of deformation of the droplet is increased and the breakup happens earlier. This behavior of the droplet is expected, because at higher
Eotvos numbers surface tension force is smaller and gravitational forces are dominant, and results in high deformation of the droplet and eventually breakup. Figures 11 and 12 show different shapes of the droplet at four selected times on the $\mathrm{Oh}_{\mathrm{d}}=.074$, for Figure 11 and 12 the Eo $=48$ and 72, respectively.

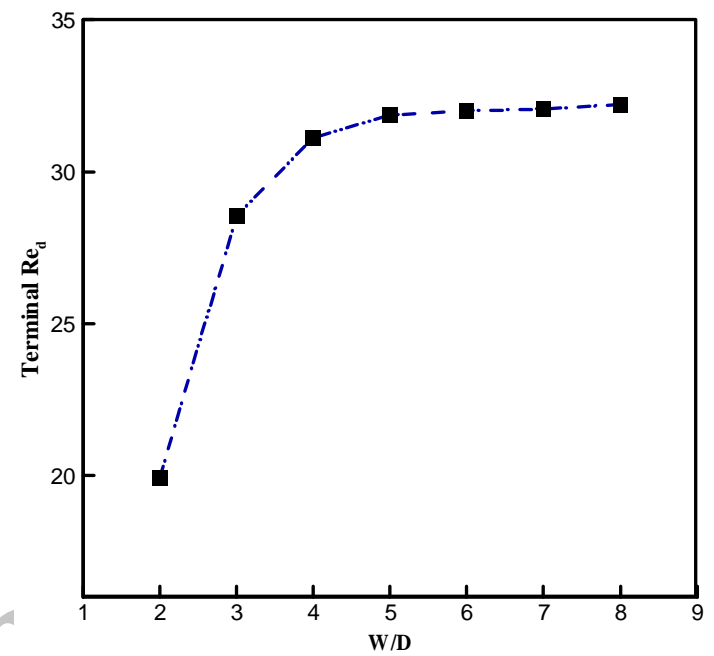

Figure 9. Terminal Reynolds number for all above simulations with different ratios of W/D.

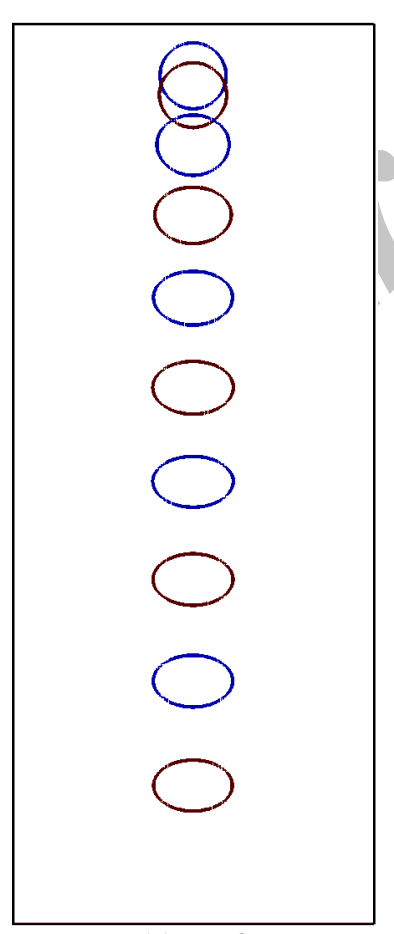

(a) $\mathrm{Eo}=2$

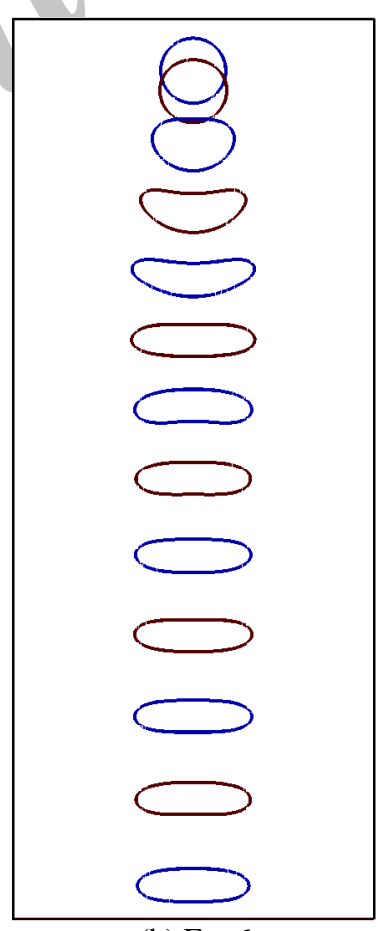

(b) $\mathrm{Eo}=6$

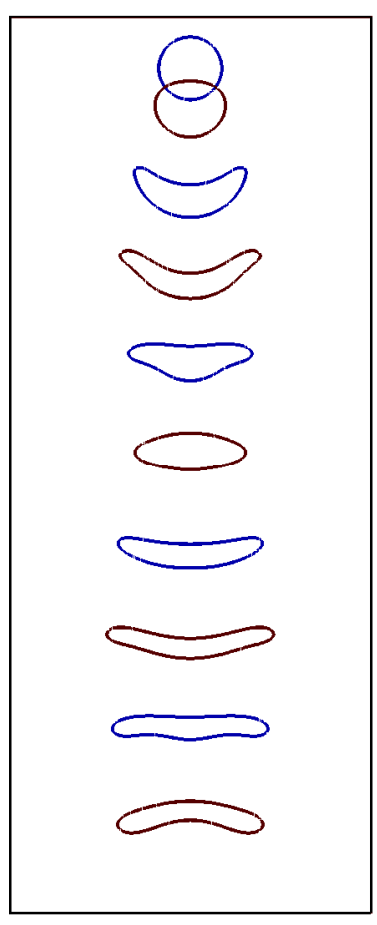

(c) $\mathrm{Eo}=12$ 


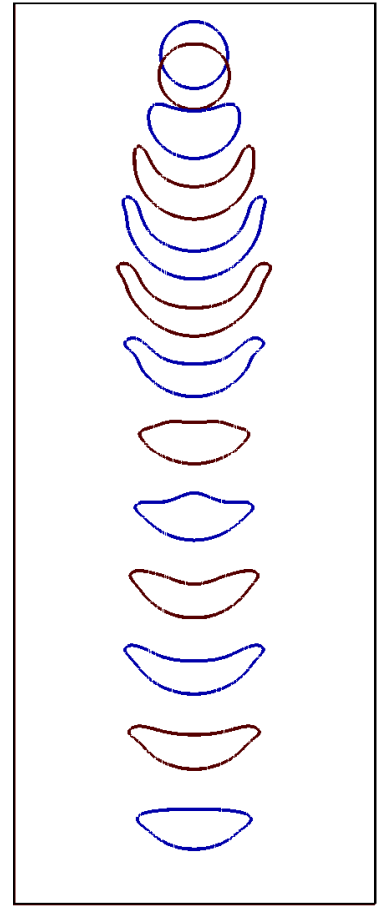

(d) $\mathrm{Eo}=24$

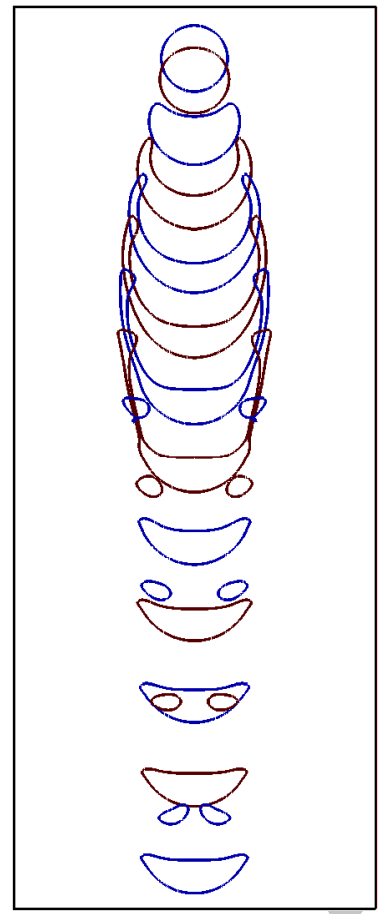

(e) $\mathrm{Eo}=48$

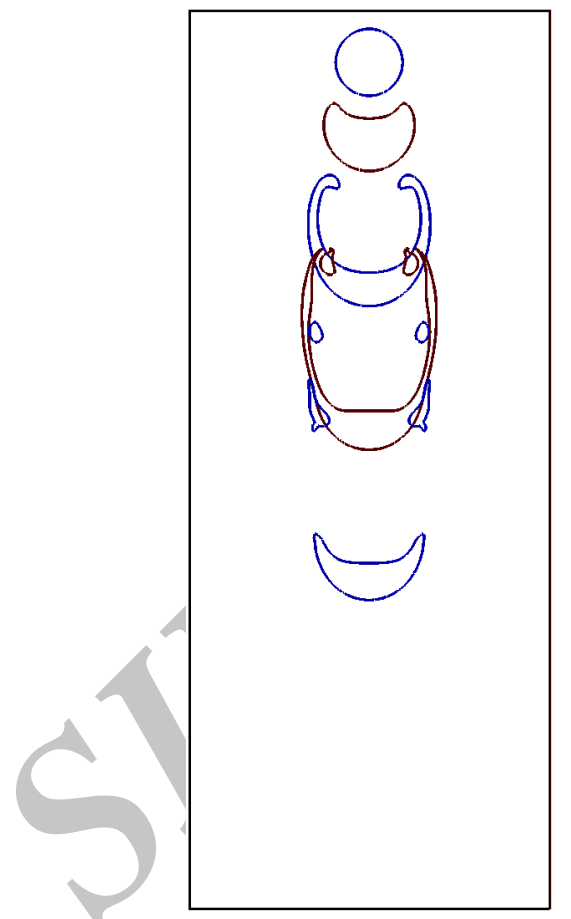

(f) $\mathrm{Eo}=72$

Figure 10. Snapshots of deformation of a falling droplet at a fixed Ohnesorge number equal to 0.074 and 0.037 for droplet fluid and surrounding fluid, respectively (the first shape in each frame corresponds to $t^{*}=0$ and for all cases except for the case (f) $\left(\Delta t^{*}=1\right)$. final dimensionless time for all cases are: $(a) t_{f}^{*}=9,(b) t_{f}^{*}=12,(c) t_{f}^{*}=9,(d) t_{f}^{*}=12,(e) t_{f}^{*}=12,(f) \Delta t^{*}=2, t_{f}^{*}=8$.

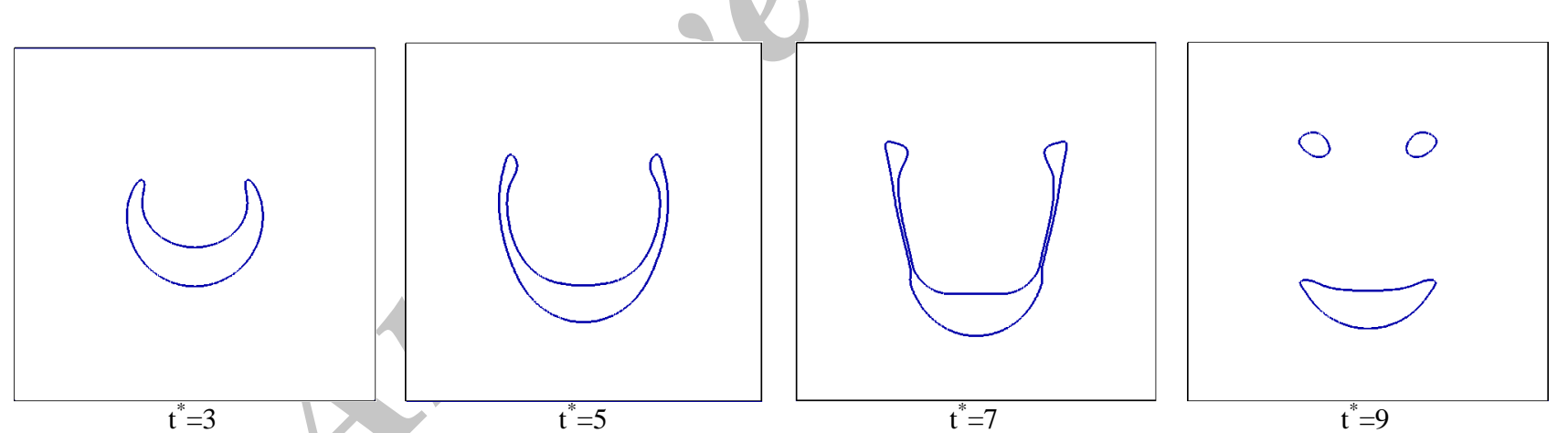

Figure 11. Different shapes for the droplet in Figure 9e, Eo $=48, \mathrm{Oh}_{\mathrm{d}}=.074, \mathrm{Oh}_{\mathrm{c}}=0.037$. The results are shown for four selected times.
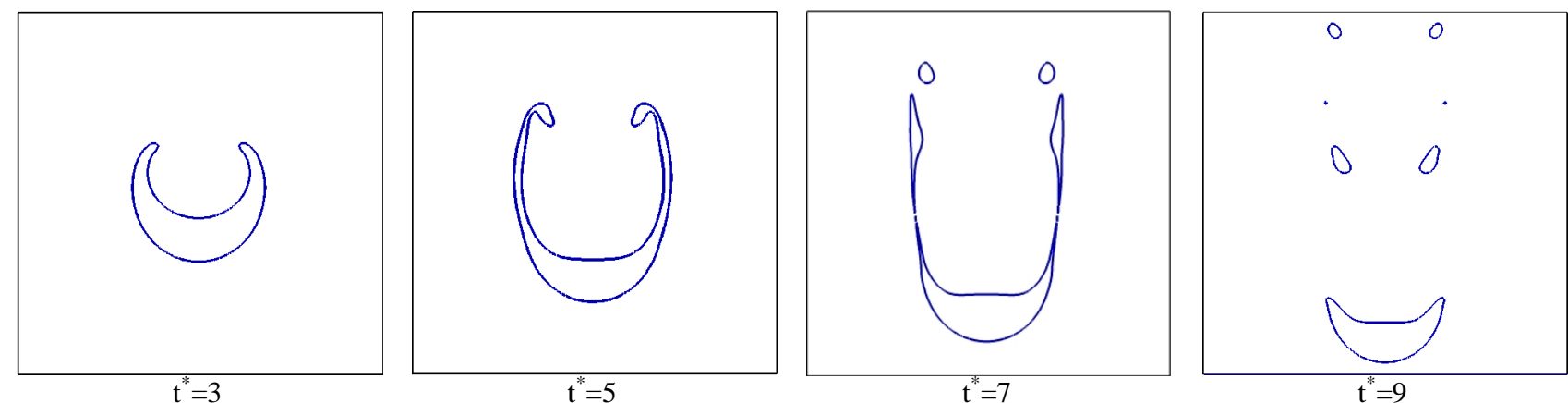

Figure 12. Different shapes for the droplet in Figure 9f, $\mathrm{Eo}=72, \mathrm{Oh}_{\mathrm{d}}=.074, \mathrm{Oh}_{\mathrm{c}}=0.037$. The results are shown for four selected times 


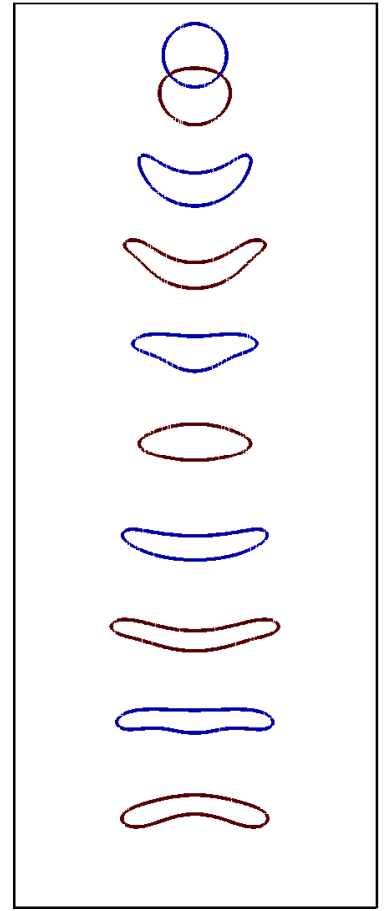

(a)

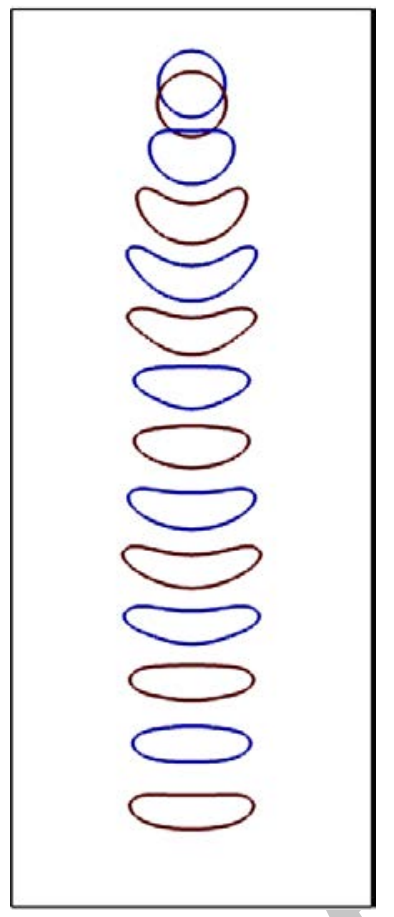

(b)

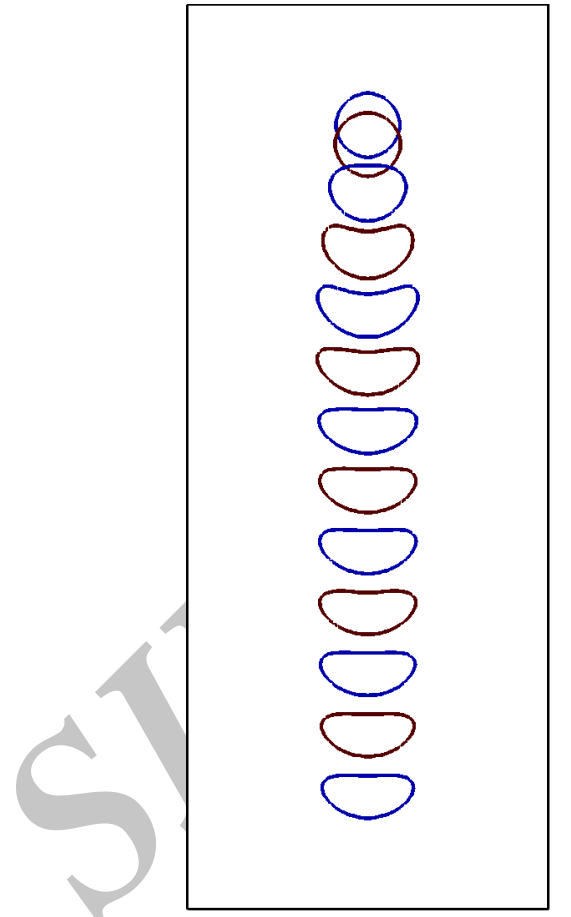

(c)

Figure 13. Snapshots of deformation of a falling droplet at a fixed Eotvos number $($ Eo $=12)$. (the first shape in each frame corresponds to $\mathrm{t}^{*}=0$ and for all cases $\Delta t^{*}=1$ ) (a) $\mathrm{Oh}_{\mathrm{d}}=0.074, \mathrm{Oh}_{\mathrm{c}}=0.037, t_{f}^{*}=9$. (b) $\mathrm{Oh}_{\mathrm{d}}=0.105, \mathrm{Oh}_{\mathrm{c}}=0.053, t_{f}^{*}=13$. (c) $\mathrm{Oh}_{\mathrm{d}}=0.15, \mathrm{Oh}_{\mathrm{c}}=0.074, i_{f}^{*}=9$
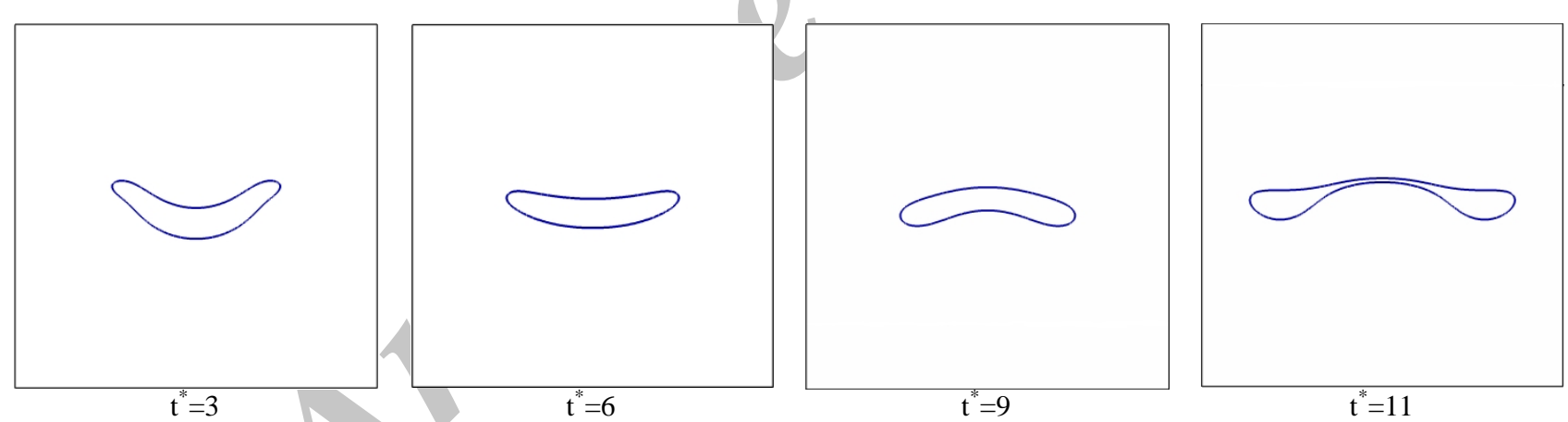

Figure 14. Different shapes for the droplet in Figure 12a, $\mathrm{Eo}=12, \mathrm{Oh}_{\mathrm{d}}=.074, \mathrm{Oh}_{\mathrm{c}}=0.037$. The results are shown for four selected times.

4. 4. Effect of Ohnesorge Number To study the effect of Ohnesorge number, Eotvos number which is held fix at 12 and $\mathrm{Oh}_{\mathrm{d}}$ is varied from 0.074 to 0.105 by changing droplet diameter (so the width of channel is increased), following that, $\mathrm{Oh}_{c}$ will also change. Figure 13 illustrates the effect of the Ohnesorge number (the non-dimensional viscosity) for droplets with a finite density ratio. As shown in Figure 13a (the same Figure $10 \mathrm{c})$, for lowest Ohnesorge number $\left(\mathrm{Oh}_{\mathrm{d}}=0.074\right)$ when Eotvos number is 12 , the droplet deforms and eventually forms a backward-facing bag and finally breakup. The initial deformation of all three droplets in Figure 13 is similar, the other two droplets in Figure
$13 \mathrm{~b}$ and $13 \mathrm{c}$ reach a steady state shape, of those, the less viscous droplet (see Figure 13b) is flatter. In Figures 14, 15 and 16, the droplets are shown at several times in separate frames. Here, $\mathrm{Oh}_{\mathrm{d}}$ is equal to $0.074,0.105$ and 0.15 , respectively, and for all cases Eotvos number is 12. These Figures show, as Ohnesorge number slightly increases, it can be seen that the rate of deformation is decreased and the droplet exhibits more resistance to the gravitational forces. It can be seen that (see Figure 13ac) the rate of deformation of the droplet is further reduced and the droplet tends to maintain its original shape. 

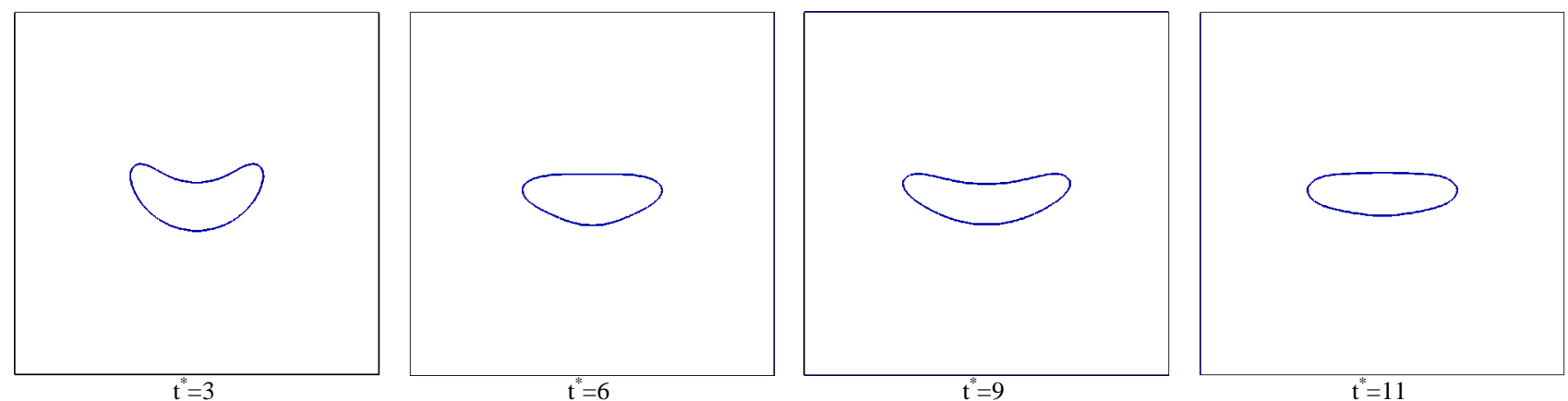

Figure 15. Different shapes for the droplet in Figure $12 \mathrm{~b}$, Eo $=12, \mathrm{Oh}_{\mathrm{d}}=0.105, \mathrm{Oh}_{\mathrm{c}}=0.053$. The results are shown for four selected times
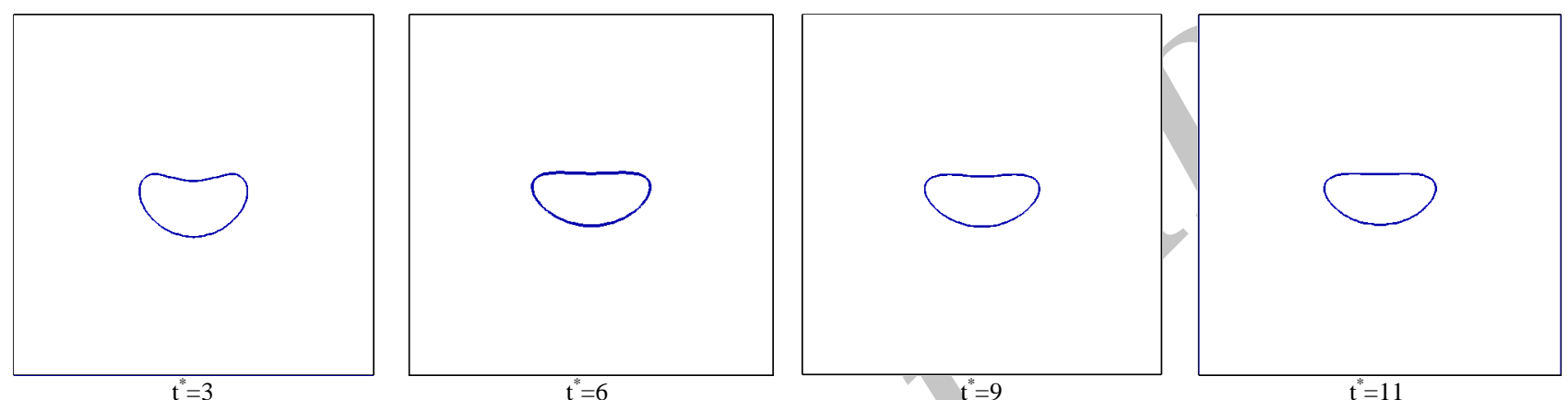

Figure 16. Different shapes for the droplet in Figure 12c, Eo $=12, \mathrm{Oh}_{\mathrm{d}}=.15, \mathrm{Oh}_{\mathrm{c}}=0.074$. The results are shown for four selected times.

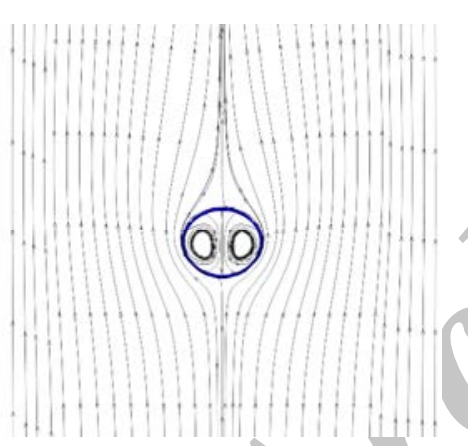

(a)

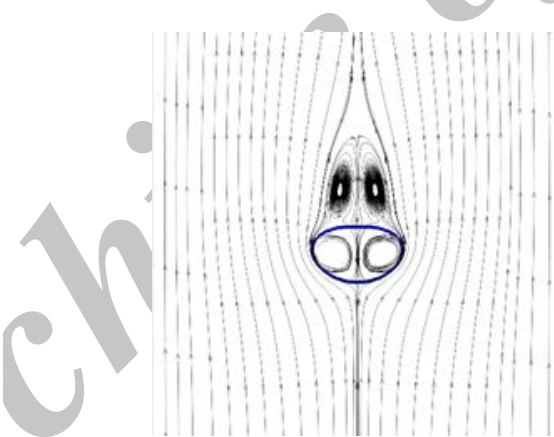

(b)

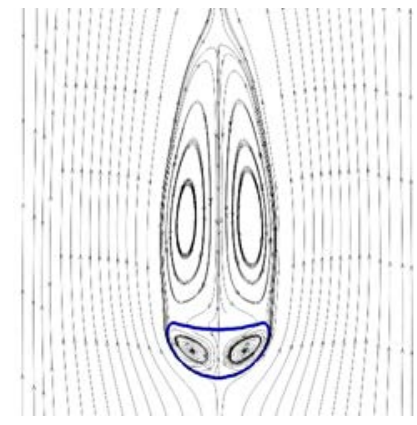

(c)

Figure 17. Streamlines around the falling droplet at three different Eotvos numbers. For all cases $\mathrm{Oh}=0.15$ and $\mathrm{t}^{*}=10$. (a) Eo=2, (b) $\mathrm{Eo}=6$, (c) $\mathrm{Eo}=24$.

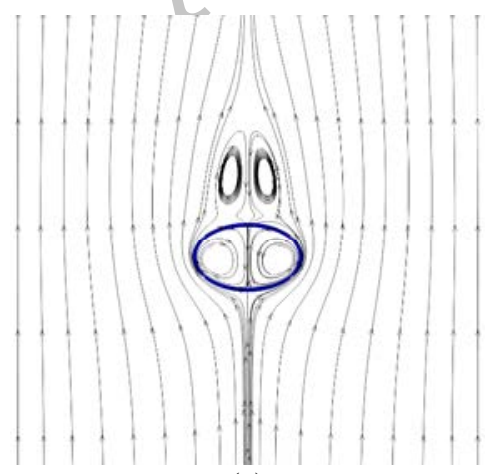

(a)

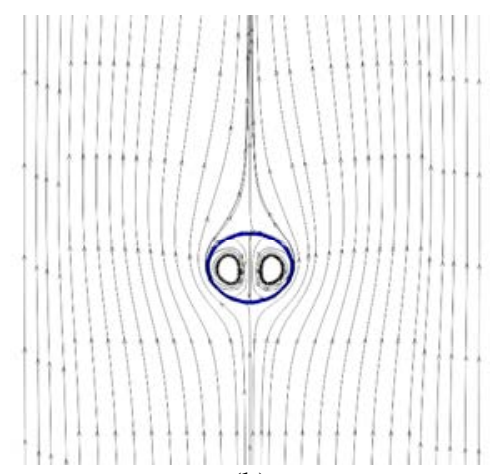

(b)

Figure 18. Streamlines around the falling droplet at two different Ohnesorge numbers. For both two cases Eo=2 and $t^{*}=6$. (a) $\mathrm{Oh}=0.074$, (b) $\mathrm{Oh}=0.15$. 
In other words, the degree of deformation of the droplet is smaller at higher Ohnesorge numbers. At higher Ohnesorge numbers, the droplet is more viscous and the viscous forces hinder the intense deformation of the droplet. Based on the results shown in Figures 13-16, it is clear that increasing both $\mathrm{Oh}_{\mathrm{d}}$ and $\mathrm{Oh}_{\mathrm{c}}$ simultaneously results in a translation of the boundaries between the breakup modes to higher Eotvos number.

4. 5. Streamlines Finally, attractive wake flow and circulations inside and above of the falling droplet are shown in Figure 17. It can be seen that with the increasing Eotvos number, surface tension effect and subsequently the viscous damping are decrease. Thus, vortex ring becomes more complex. As shown in Figure $17 \mathrm{a}-\mathrm{c}$, the increasing effect of the Eotvos number reduces the stability of the vortex and circulations in the wake flow. It is observed in Figure 17a that the vortices are created inside the droplet, and as shown in Figure $17 \mathrm{~b}$ with increasing Eotvos number, vortices also created inside the flow field and above the droplet. Furthermore, figure 17c shows that for highest Eotvos number $($ Eo $=24)$, vortices created inside the flow field becomes larger. The external flow field is not also laminar compared with lower Eotvos numbers.

As shown in Figure 18, the effect of increasing Ohnesorge number at fixed Eotvos number on the circulations is same as decreasing Eotvos number at fixed Ohnesorge number.

\section{CONCLUTION}

In this study, the deformation and breakup of a 2D falling droplet, accelerated by gravity force have been studied by lattice Boltzmann method. In addition, for some range of Eotvos and Ohnesorge numbers which were simulated for low Ohnesorge numbers $(\mathrm{Oh}<0.1)$, the Eotvos number is the main controlling parameters. At very low Eotvos numbers, the droplet deforms, but does not breakup. For Eotvos number around 12, the droplet breaks up by the formation of a backward facing bag. For Eotvos number around 24, the drop moves with an essentially steady convex shape, showing no sign of bag formation, and for high Eotvos numbers (Eo>45 in this paper) shear breakup mode is observed. As Oh is increased, the effect of the viscosity reduces the rate of deformation. The simulations show that the main effect of increasing Ohnesorge number is to move the boundary between the different breakup modes to higher Eotvos number.

\section{REFERENCES}

1. Fakhri, A. and Rahimian, M. H. "Investigation of deformation and breakup of a falling droplet using a multiple-relaxation- time lattice Boltzmann method", Journal of. Computers \& Fluids, Vol. 40, (2011), 156- 171.

2. Fakhri, A., and Rahimian, M. H., "Simulation of falling droplet by the lattice Boltzmann method", Communications in Nonlinear Science and Numerical Simulation, Vol. 14, (2009), 3045-3046.

3. Magarvey, R H. and Taylor, B W., "Free fall breakup of large drops", Journal of Applied Physics, Vol. 27, (1956), 1129-1135.

4. Kojima, M. Hinch, EJ. and Acrivos A., "The formation and expansion of a toroidal drop moving in a viscous fluid", Physics of Fluids, Vol. 27, (1984), 19-32.

5. Ni, M J. Komori, S. and Morley, N B., "Direct simulation of falling droplet in a closed channel", International Journal of Heat and Mass Transfer, Vol. 49, (2006), 366-376

6. Feng, J.Q., "A deformable liquid drop falling through a quiescent gas at terminal velocity", Journal of Fluid Mechanics, Vol. 658, (2010), 438-462.

7. Han, J. and Tryggvason G., "Secondary breakup of axisymmetric liquid drops, I Acceleration by a constant body force", Physics of Fluid, Vol. 11, (1999), 3650-3660

8. Jalaal, M. Mehravaran, K., "Fragmentation of falling liquid droplets in bag breakup mode", International Journal of Multiphase Flow, Vol. 47, (2012), 115-132.

9. Frisch, U., Hasslacher, B. and Pomeau, Y., "Lattice gas automata for Navier-Stokes equation", Physical Review Letters, Vol. 56, (1986), 1505-1508.

10. Chen, H. Chen, S. and Matthaeus, W. H., "Recovery of the Navier-Stokes equations using a lattice-gas Boltzmann method", Physical Review A, Vol. 45, (1992) 5339-5342

11. Hasanzadeh Afrouzi, H. Sedighi, M. Farhadi, M. and Fattahi, E. "Dispersion and deposition of micro particles over two square obstacles in a channel via hybrid lattice Boltzmann method and discrete phase medel", International Journal of Engineering, Vol. 25, (2012), 257-266.

12. Jafari, M. Farhadi, M. Sedighi, K. and Fattahi, E., "Effect of wavy wall on convection heat transfer of water-A12o3 nanofluid in a lid driven cavity using lattice Boltzmann method", International Journal of Engineering, Vol. 25, (2012), 165-176

13. Abouali, O. and Ahmadi, G., "Three-dimensional simulation of airflow and nano-particle beam focusing in aerodynamic lenses", International Journal of Engineering, Vol. 20, (2007), 45-54.

14. Fathaddin, M. T. and Awang, M. B., "Lattice gass automata simulation of adsorption process of polymer in porous media", International Journal of Engineering, Vol. 17, (2004), 329338.

15. Gunstensen, A. K. and Rothman, D. H., "Lattice Boltzmann model of immiscible fluids", Physical Review A, Vol. 43, (1991), 4320-4327

16. Shan, X. Chen, H., "Lattice Boltzmann model for simulating flows with multiple phases and components", Physical Review. E., Vol. 47, (1993), 1815-1819.

17. Shan, X., "Pressure tensor calculation in a class of nonideal gas lattice Boltzmann models", Physical Review E, Vol. 77, (2008), (6 Pt 2):066702

18. Sbragaglia, M. Benzi, R. Biferale, L. Succi, S. Sugiyama, K Toschi, F., "Generalized lattice Boltzmann method with multirange pseudopotential", Physical Review E, Vol. 75, (2007).

19. Swift, M. R., Orlandini, E. Osborn, W. R. and Yeomans, J. M., "Lattice Boltzmann simulations of liquid-gas and binary fluid systems", Physical Review E, Vol. 54, (1996), 5041-5052

20. He, X. Shan, X. and Doolen, G. D., "Discrete Boltzmann equation model for non ideal gases", Physical Review E, Vol. 57, (1998).

21. He, X. Shan, X. and Doolen, G. D., " A novel thermal model for the lattice Boltzmann method in incompressible limit", Journal of Computational Physics, Vol. 146, (1998), 282-300 
22. Shan, X. and Doolen, G., "Multi-component lattice-Boltzmann model with interparticle interaction", Journal of. Statistical. Physics, Vol. 81, (1995), 379-385.

23. Qian, Y. H., D'Humieres, D. and Lallemand, P., "Lattice BGK Models for Navier-Stokes Equation", Europhysics Letters, Vol. 17, (1992), 479-484.

24. Gupta, A. Kumar, R., "Lattice Boltzmann simulation to study multiple bubble dynamics", International Journal of Heat and Mass Transfer, Vol. 51, (2008), 5192-5203.

25. Zhao, Y., "A novel lattice Boltzmann method for direct numerical simulation of multiphase slows", Ohio State University Thesis, (2009).

26. Ngachin, M., "Simulation of rising bubbles dynamics using the lattice Boltzmann method", Florida International University, Thesis, (2011).

27. Martys, N. S. and Chen, H., "Simulation of multicomponent fluids in complex three-dimensional geometries by the lattice Boltzmann method", Physical Review E, Vol. 53, (1996), 743

28. Shan, X. and Chen, H., "Simulation of non ideal gasses and liquid-gas phase transitions by the lattice Boltzmann equations", Physical Review E., Vol. 49, (1994), 2941-2948
29. Huang, H., Thorne, D. T., Schaap, M. G. and Sukop, M. C., "Proposed approximation for contact angles in the Shan-andChen-type multicomponent multiphase lattice Boltzmann models", Physical Review E, Vol. 76, (2007), 1-6

30. He, X. and Doolen, G., "Thermodynamic foundations of kinetic theory and lattice Boltzmann models for multiphase flows", Journal of Statistical Physics, Vol. 107, (2002), 309-328.

31. Gong, S., Cheng, P., and Quan, X., "Lattice Boltzmann simulation of droplet formation in microchannels under an electric field", International Journal of Heat and Mass Transfer, Vol. 53, (2010), 5863-5870

32. Yang, Z. L., Dinh, T. N., Nourgaliev, R. R. and Sehgal, B. R., "Numerical Investigation of bubble growth and detachment by the lattice-Boltzmann method", International Journal of Heat and Mass Transfer, Vol. 44, (2001), 195- 206.

33. Sukop, M. C. and Thorne, D. T., "Lattice Boltzmann modeling an introduction for geoscientists and engineers", Berlin: Springer, (2005)

34. Annaland, M. S., Deen, N. G., and Kuipers, J. A. M. "Numerical simulation of gas bubbles behaviour using a three dimensional volume of fluid method", Chemical Engineering Science, Vol. 60, (2005), 2999 - 3011.

\title{
Lattice Boltzmann Simulation of Deformation and Breakup of a Droplet under Gravity Force Using Interparticle Potential Model
}

\author{
S. E. Mousavi Tilehboni, K. Sedighi, M. Farhadi, E. Fattahi
}

Faculty of Mechanical Engineering, Babol Noshirvani University of Technology, Postal Code 4714871167, Babol, Iran

PAPER INFO

\section{Paper history:}

Received 16 January 2013

Received in revised form 17 February 2013

Accepted 28 February 2013

\section{Keywords:}

Falling droplet

Breakup

Lattice Boltzmann method

Interparticle potential model

Eotvos number

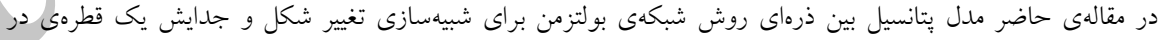

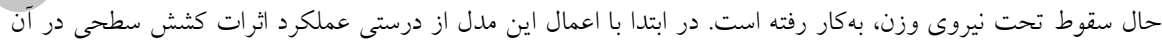

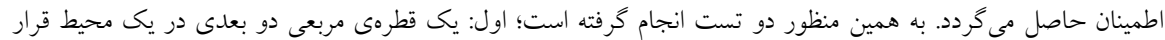

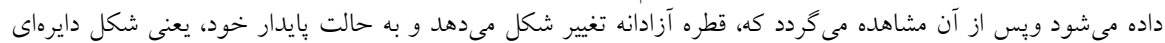

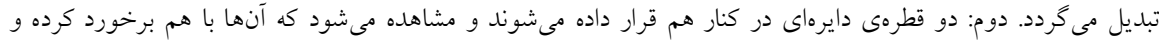

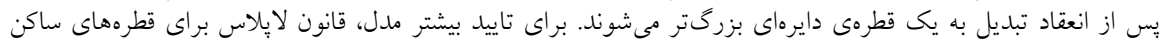

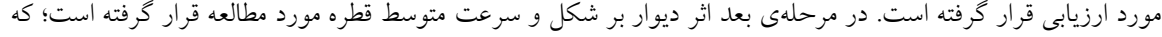

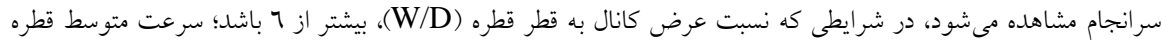

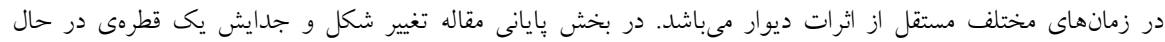

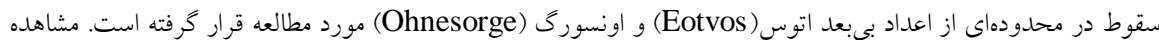

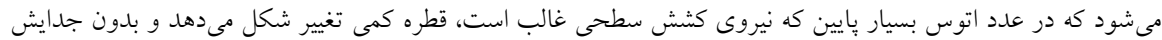

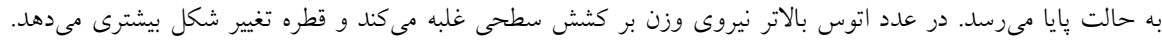

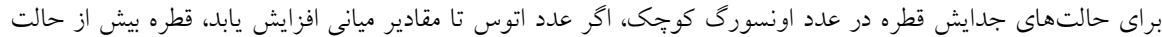

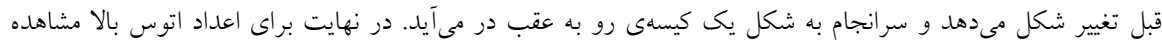

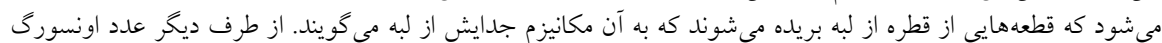

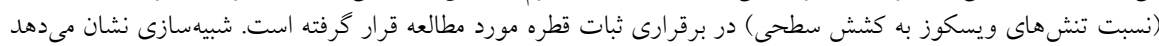

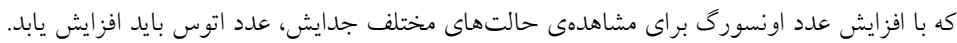




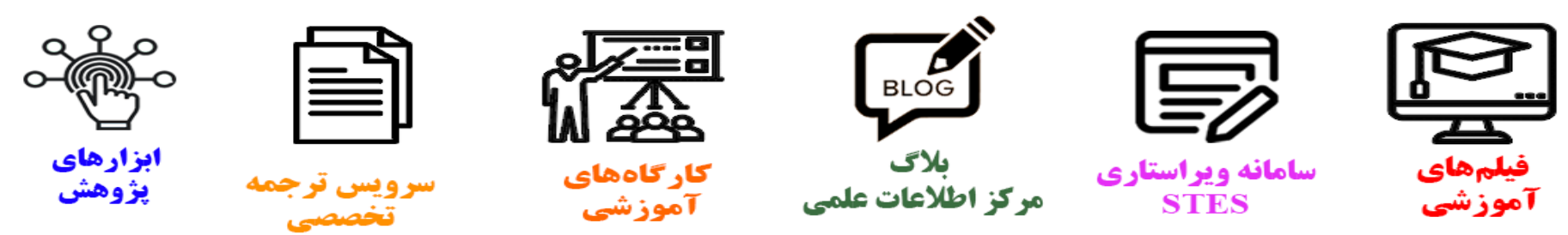

\section{(c)}

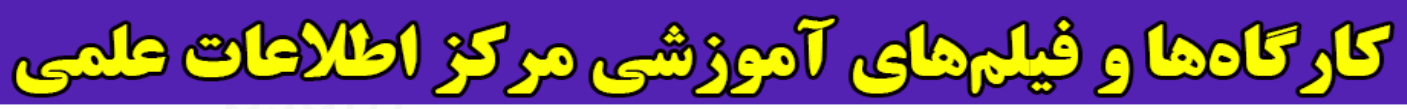
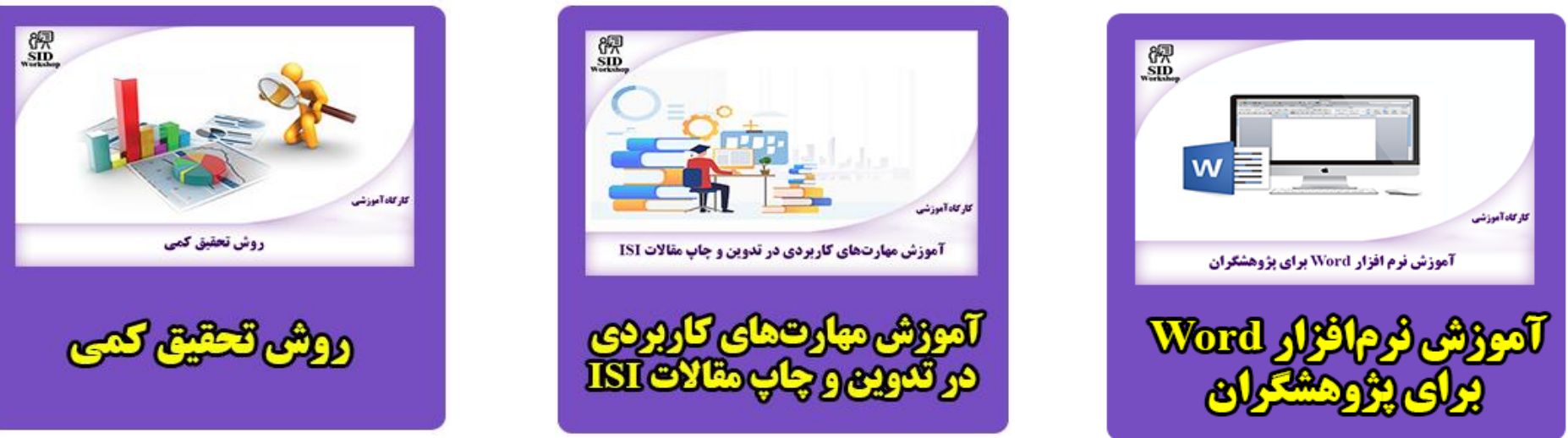